\title{
Energy Household Forecast with ANN for Demand Response and Demand Side Management
}

\author{
Filipe Rodrigues $^{1,2}$, Carlos Cardeira ${ }^{3}$, J.M.F.Calado ${ }^{1,3}$, R. Melício ${ }^{3,4}$ \\ ${ }^{1}$ Instituto Superior de Engenharia de Lisboa, Instituto Politécnico de Lisboa \\ Departamento de Engenharia Mecânica. \\ Rua Conselheiro Emídio Navarro, 1959-007 Lisbon, Portugal \\ e-mail: fmrodrigues@dem.isel.pt \\ ${ }^{2}$ MIT Portugal, Campus IST - Tagus Park \\ Av. Professor Cavaco Silva, 2744-016 Porto Salvo, Portugal \\ e-mail: fmrodrigues@ dem.isel.ipl.pt \\ ${ }^{3}$ IDMEC/LAETA, Instituto Superior Técnico, Universidade de Lisboa \\ Av. Rovisco Pais, 1049-001 Lisbon, Portugal \\ e-mail: carlos.cardeira@ist.utl.pt \\ ${ }^{4}$ Departamento de Física, Escola de Ciências e Tecnologia, Universidade de Évora, Portugal \\ ruimelicio@gmail.com
}

\begin{abstract}
This paper presents a short term load forecasting with artificial neural networks. Despite the great imprevisibility, it is possible to forecast the electricity consumption of a household with some accuracy, similarly to that the electricity utilities can do to an agglomerate of households. Nowadays, in an existing electric grid, it is important to understand and forecast household daily or hourly consumption with a reliable model for electric energy consumption and load profile. Demand response programs required this information to adequate the profile of energy load diagram to generation. In the short term load forecasting model, artificial neural networks were used, with a consumption records database. The results show that the artificial neural networks approach provides a reliable model for forecasting household electric energy consumption and load profile. To do so and using smart devices such as cyber-physical systems monitoring, gathering and computing in real time a database with weekdays and weekend, can improve forecasts results for the next hours, a strong tool for Demand Response and Demand Side Management.
\end{abstract}

\section{Key words}

Demand Side Management, Demand Response, ANN, Household, Energy, Forecast.

\section{Introduction}

An important approach to demand response and energy management to promote a more efficient energy end user is Demand Side Management [1]. This approach includes the transfer of some units for the periods when demand is lower and energy storage to greater supply (production). Through knowledge of the profile curve to power consumption of each household it is possible to find a model for optimizing the electrical energy used [2].
The aim of this paper is to show the applicability of an Artificial Neural Network (ANN) approach to develop a simple and reliable method to forecast households' daily and hourly energy consumption for residential and small buildings. ANN favored as a method for prediction in many applications. The models achieved using ANN are inspired on the information management process of the human brain which formulates a solution through the use of previously accumulated data [3]. The ANN have been trained and tested using energy consumption data obtained from direct measurement in households. A database with consumption records measured between February 2000 and July 2001 of 93 households, in Portugal, and that was used by O. Sidler's research team [4], has been used during the current studies. After a hard work to select and accept the valid data, all of the daily and hourly energy consumption values of one and half year were used in training and testing the model.

The technical literature is abundant with techniques and approaches for performing or improving STLF. A number of approaches work well with certain power systems or certain geographical areas, while they fail for some other systems due to the nature of the electric load demand: it is complex, highly nonlinear, and dependent on weather, seasonal and social factors.

A number of researchers have compiled extensive surveys on load forecasting. Some of these surveys have been focused on neural networks for STLF [4], [5], some other techniques used such as time series and regression models [6], as well as approaches based on exponentially weighted methods [7], while some other authors provided a general look at all types of load forecasting methodologies [8]. ANN has received a large share of 
attention and interest.

The review of literature mentioned in this paper enabled us to establish in our research a number of variables that are based on the rise in energy consumption patterns by households, both during weekdays and weekend, and use the ANN to make forecast the typical values for energy consumption per household for one day, considering a specific type of household.

This paper focuses on short term load forecasting with artificial neural networks. Despite the great imprevisibility, it is possible to forecast the electricity consumption of a household with some accuracy, similarly to that the electricity utilities can do to an agglomerate of households. The rest of the paper is organized as follows. Section 2 presents the methodology. Section 3 presents the results and discussion Finally, concluding remarks are given in Section 4.

\section{Methodology}

Selecting an appropriate architecture is the first step to take when designing an ANN based forecasting system. The network architecture was built based on multilayer perceptron (MLP), full-connected, which is a feedforward type of artificial neural network, and the training task was performed through a backpropagation learning algorithm.

The backpropagation learning algorithms are based on steepest-descent methods that perform stochastic gradient descent on the error surface. The output layer is then analyzed to see the contribution of each of the neurons to that error. The neurons weights and threshold values are then adjusted, according to how much each neuron contributed to the error, to minimize the error in the next iteration.

This study used the learning Levenberg-Marquardt (LM) algorithm (Levenberg, 1944 and Marquardt, 1963) that works as a training algorithm with the capabilities of the pruning methodologies. By doing the artificial neural network prune the model achieved has reduced complexity and the computational effort to run it, especially in real time, is reduced too.

The Levenberg-Marquardt algorithm, which consists in finding the update given by

$\Delta x=-\left[J^{T}(x) J(x)+\mu I\right]^{-1} J^{T}(x) e(x)$

where $J(x)$ is the Jacobian matrix, $\mu$ is a parameter conveniently modified during the algorithm iterations and $e(x)$ is the error vector. When $\mu$ is very small or null the LM algorithm becomes Gauss-Newton, which should provide faster convergence, however for higher $\mu$ values, when the first term within square brackets of (1) is negligible with respect to the second term within square brackets, the algorithm becomes steepest descent. Hence, the LM algorithm provides a nice compromise between the speed of Gauss-Newton and the guaranteed convergence of steepest descent [9].

Furthermore, the output of any neuron is shown in Fig. 1.

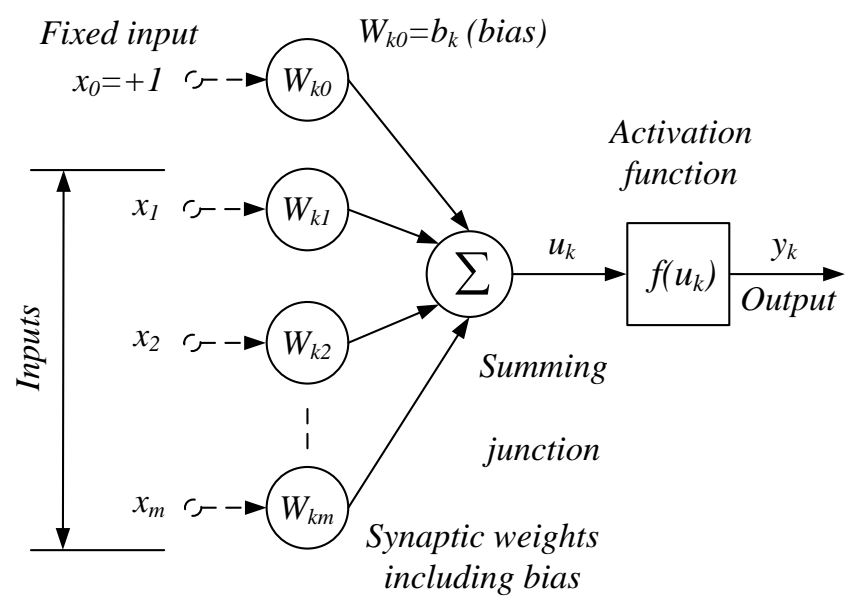

Fig. 1. Perceptron neurons.

The output of an artificial neuron, mathematically, is given by

$$
y_{k}=f\left(u_{k}\right)=f\left(\sum_{j=1}^{m} w_{k j} x_{j}+b_{k}\right)
$$

or considering bias as input value $x_{0}=1$ and weight $w_{k 0}=b_{k}$ given by

$$
y_{k}=f\left(u_{k}\right)=f\left(\sum_{j=0}^{m} w_{k j} x_{j}\right)
$$

The transfer function $f$ can be selected from a set of readily available functions.

The MLP used in the current studies has three layers: an input layer, a hidden layer and an output layer. The number of neurons used in the input layer depends on the number of input variables being considered as inputs of the forecasting model, while the number of neurons in the output layer depends on the number of variables that one wants to predict with a specific model.

The activation function for the hidden and output neurons must be differentiable and non-decreasing [10]: the current studies has been adopted the linear and the hyperbolic tangent functions. The activation functions are used to scale the output given by the neurons in the output layer.

The ANN architecture was built with three-layer feedforward configuration. In order to achieve the desired performance for the forecasting model obtained, as well as during the artificial neural network training stage, following a trial and error procedure, the network was optimized as mentioned in [2].

In order to forecast the hourly energy consumption for a usual day, as mentioned above, it was used a network which is shown in Fig. 2. 


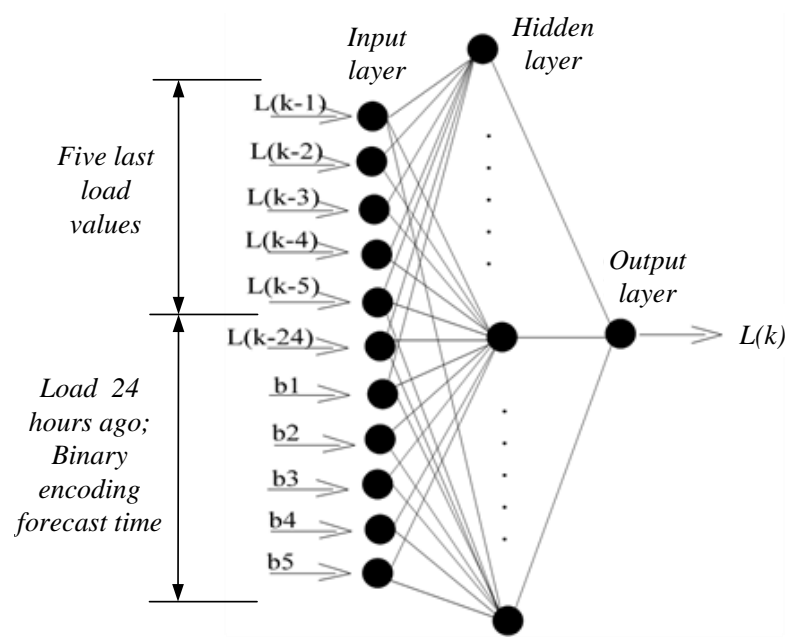

Fig. 2. Simplified ANN architecture used to forecast hourly load.

The inputs of the network were the hourly energy consumption of each household, including both weekdays and weekend and the output was the next hourly energy consumption.

To reduce the complexity of the training task, for the hourly consumption, a total of 3 week data, i.e., 32,760 data (504 hours x 65 households $=32,760$ hours) were used. For each household it was used 504 hours, 2/3 of those data (336 hours), the two first weeks, making up the first subset, were used for ANN training and validation. The last $1 / 3$ of the original data (168 hours), the last week, making up the second subset, was used to evaluate the prediction capacity of the developed ANN model.

The ANN model used Boolean input as an hourly meter system [11] for the forecast hourly load [12]. This method uses an input vector composed of Boolean variable and can take any configuration among $2 \mathrm{n}$ different vectors containing Boolean values such as: $0000 \ldots 0=01: 00 \mathrm{~h} ; 1000 \ldots 0=02: 00 \mathrm{~h} ; 01000 \ldots 0=$ $03: 00 \mathrm{~h} ; 111 \ldots 1=24: 00 \mathrm{~h}$.

This model uses a concept of 11 data in the input layer: load energy ( 5 last hour load values and for 6th hour the load 24 hours ago) and binary encoding for the time ( 0 to 24 hours) with $n=5 ; 20$ neurons in the hidden layer and; 1 output (next hourly load) in the output layer.

The network training was performed by using LM backpropagation algorithms, Hyperbolic tangent sigmoid function (HTSF) and pure line function (PF) were used as the transfer function in the hidden layer and output layer, respectively, and PF in input layer, performed under software tool (nntool) developed by Matlab.

For this network, the forecasting correlation and error values of the outputs were calculated and compared with test set, as well the shape of the load profile distribution.

The effectiveness of this approach is confirmed by forecast outputs obtained. In conclusion, the proposed artificial neural network shows a reliable performance for load forecasting.

The evaluation of the ANN performance is based on the shape of the load profile distribution and following criterion: mean absolute percent error (MAPE); the standard deviation of error (SDE) and; serial correlation (linear correlation $\mathrm{R}$ and linear regression $\mathrm{R} 2$ ).

\section{Results and Discussion}

The ANN model used the Boolean input application, with 11 inputs, one hidden and one output layer, as shown in Fig. 2, and for learning the neural network, that was adopted the LM algorithm and a network which had 20 neurons in its hidden layer.

The ANN was trained using the first two weeks of data, one random household and tested for the next days of 3th week. Fig. 3 and Fig. 4 show the forecast hourly load of random households for the first day.

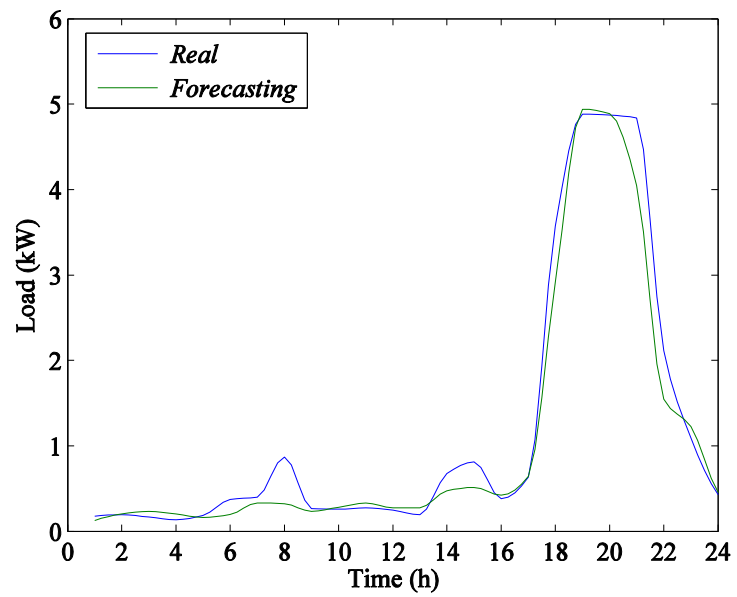

Fig. 3. Forecast Hourly load of Household $n^{\circ} 64$ - first days.

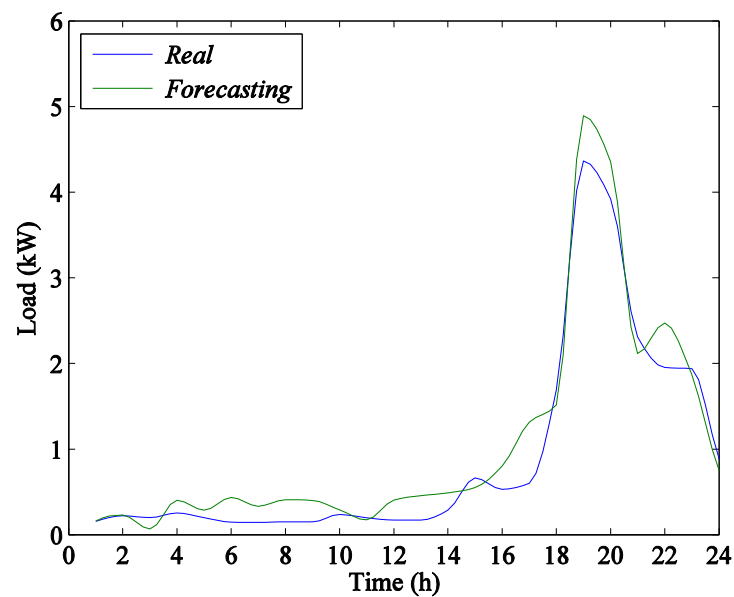

Fig. 4. Forecast Hourly load of Household n ${ }^{\circ} 65$ - first days.

In test process, the forecasting correlation and error values of the outputs were calculated are shown in Table I. 
Table I. Statistical analysis of the forecasting error attained with the neural network approach for the households H64 (left) and H65 (right) analyzed.

\begin{tabular}{ccccccccc}
\hline Day & \multirow{2}{*}{$\mathrm{R}$} & \multirow{2}{*}{$\mathrm{R}^{2}$} & $\mathrm{MAPE}$ & $\mathrm{SDE}$ & $\mathrm{R}$ & $\mathrm{R}^{2}$ & $\mathrm{MAPE}$ & $\mathrm{SDE}$ \\
\hline 1 & $98,8 \%$ & $97,7 \%$ & $16,0 \%$ & 0,23 & $98,3 \%$ & $96,6 \%$ & $21,5 \%$ & 0,17 \\
2 & $99,6 \%$ & $99,3 \%$ & $10,0 \%$ & 0,09 & $97,6 \%$ & $94,5 \%$ & $19,3 \%$ & 0,21 \\
3 & $99,3 \%$ & $98,6 \%$ & $12,9 \%$ & 0,14 & $97,9 \%$ & $93,2 \%$ & $23,5 \%$ & 0,22 \\
\hline
\end{tabular}

Evaluating those Figures and Table I it is found that for daily profiles which have a similar pattern the method proposed by [12] has a good performance for the first three days.

\section{Conclusion}

This paper introduced forecasting methods of daily and hourly energy consumption, by using an Artificial Neural Network (ANN) using an algorithm, such as LevenbergMarquardt, the research reveal that ANN are able to forecast daily and hourly energy consumption, as well the load profile with accuracy.

The paper introduced the ANN technique for modeling energy consumption for a random day and next hours, using a Boolean metering system.

Forecast hourly and daily energy consumption can be useful on the demand-side management, such as electricity suppliers, to forecast the likely future development of electricity demand in the entire sector of the community.

For future research, an important step is continuing this additional research enhancing forecasting capability on the load profile renewable energy production (micro production) to identify an accurate, effective and appropriate renewable energy production and energy storage.

\section{Acknowledgments}

This work is funded by Portuguese Foundation for Science \& Technology, through IDMEC, under LAETA, project UID/EMS/50022/2013.

\section{References}

[1] R.Pereira, R. Melicio, V.M.F. Mendes, J. Figueiredo, A. Fagundes, and J.C. Quadrado, "Fuzzy clustering applied to a demand response model in a smart grid contingency scenario", in: International Power Electronics, Electrical Drives, Automation and Motion, 495-499, Ischia, Italy, 2014.
[2] F. Rodrigues, C. Cardeira, and J.M.F. Calado, "The daily and hourly energy consumption and load forecasting using artificial neural network method: a case study using a set of 93 households in Portugal", Energy Procedia, vol. 62, pp. 220-229, 2014.

[3] I.H. Yang, and K.W. Kim, "Prediction of the time of room air temperature descending for heating systems in buildings", Building and Environment, vol. 39, pp. 19-29, 2004.

[4] Project Eureco, "Demand side management. End-use metering campaign in 400 households of the European Community", in: SAVE Programme, Commission of the European Communities, 2002.

[5] O. Carpinteiro, A. Reis, and A. Silva, "A hierarchical neural model in short-term load forecasting", Applied Soft Computing, vol. 4, pp. 405-412, 2004.

[6] G. Gross, and F. Galiana, "Short-term load forecasting", Proceedings of IEEE, vol. 75, pp. 1558-1573, 1987.

[7] J. Taylor, "Short-term load forecasting with exponentially weighted methods", IEEE Transactions on Power Systems, vol. 27, pp. 458-464, 2012.

[8] E. Feinberg, and D. Genethliou, "Load forecasting. Applied mathematics for restructured electric power systems", in: Optimization, Control, and Computational Intelligence, Springer, pp. 269-285, 2005.

[9] L. Saini, and M. Soni, "Artificial neural network based peak load forecasting using Levenberg-Marquardt and quasi-Newton methods", in: IEEE Generation, Transmission and Distribution, vol. 149, 578-584, 2002.

[10] H. Hippert, C. Pedreira, and R. Souza, "Neural networks for short-term load forecasting: a review and evaluation", IEEE Transactions on Power Systems, vol. 16, pp. 44-55, 2001.

[11] W. Holderbaum, R. Canart, and P. Borne, "Artificial neural networks application to boolean input systems control", Studies in Informatics and Control, vol. 8, pp. 107-120, 1999.

[12] R.S. Zebulum, M. Vellasco, K. Guedes, and M.A. Pacheco, "Short-term load forecasting using neural nets", in: Natural to Artificial Neural Computation, pp. 10011008, 1995. 